\title{
A Fast and Parametric Torque Distribution Strategy for Four-Wheel-Drive Energy-Efficient Electric Vehicles
}

\author{
Arash M. Dizqah, Member, IEEE, Basilio Lenzo, Member, IEEE, Aldo Sorniotti, Member, IEEE, \\ Patrick Gruber, Saber Fallah, and Jasper De Smet
}

\begin{abstract}
Electric vehicles (EVs) with four individually controlled drivetrains are over-actuated systems, and therefore, the total wheel torque and yaw moment demands can be realized through an infinite number of feasible wheel torque combinations. Hence, an energy-efficient torque distribution among the four drivetrains is crucial for reducing the drivetrain power losses and extending driving range. In this paper, the optimal torque distribution is formulated as the solution of a parametric optimization problem, depending on the vehicle speed. An analytical solution is provided for the case of equal drivetrains, under the experimentally confirmed hypothesis that the drivetrain power losses are strictly monotonically increasing with the torque demand. The easily implementable and computationally fast wheel torque distribution algorithm is validated by simulations and experiments on an EV demonstrator, along driving cycles and cornering maneuvers. The results show considerable energy savings compared to alternative torque distribution strategies.
\end{abstract}

Index Terms - Control allocation (CA), electric vehicle (EV), experiments, power loss, torque distribution.

\section{INTRODUCTION}

$\mathbf{O}$ $\mathrm{NE}$ of the main obstacles to the success of electric vehicles $(E V s)$ in the automotive market is their limited driving range. This issue is addressed by research in novel battery technologies to increase energy density and, hence, to provide viable/lightweight high-capacity energy storage systems. On the other hand, energy management systems are conceived to improve vehicle efficiency through advanced control of the drivetrains and ancillaries. In particular, EVs with multiple drivetrains allow the implementation of control functions, such as front-to-rear and left-to-right torque vectoring,

Manuscript received August 10, 2015; revised December 1, 2015; accepted February 2, 2016. This work was supported by the European Union Seventh Framework Programme FP7/2007-2013 under the iCOMPOSE project under Grant 608897.

A. M. Dizqah was with the Centre for Automotive Engineering, University of Surrey, Guildford, GU2 7XH, U.K. He is now with the Centre for Mobility and Transport, Coventry University, Coventry, CV1 5FB, U.K. (e-mail: arash.moradinegadedizqah@ coventry.ac.uk).

B. Lenzo, A. Sorniotti, P. Gruber, and S. Fallah are with the Centre for Automotive Engineering, University of Surrey, Guildford, GU2 7XH, U.K. (e-mail: b.lenzo@ surrey.ac.uk; a.sorniotti@ surrey.ac.uk; p.gruber@surrey.ac.uk; s.fallah@surrey.ac.uk).

J. De Smet is with Flanders MAKE, 3920 Lommel, Belgium (e-mail: jasper.desmet@flandersdrive.be).

Color versions of one or more of the figures in this paper are available online at http://ieeexplore.ieee.org.

Digital Object Identifier 10.1109/TIE.2016.2540584 which improve active safety and drivability, and contribute to the attractiveness of EV technology [1], [2]. Owing to the use of multiple motors, an actuation redundancy is obtained, i.e., the desired vehicle behavior corresponding to the driver's inputs at the accelerator/brake pedal and/or steering wheel can be realized through an infinite number of feasible wheel torque distributions. For the range of feasible torque distributions, this paper presents a novel solution enhancing energy efficiency.

The two major sources of power loss in EVs are the drivetrains and the tires. The drivetrain power losses include the contributions of the drives, electric motors, and transmissions (if present). Tire contributions relate to rolling resistance, longitudinal slip, and lateral slip, with the latter two being relevant only at significant acceleration levels [3].

Prior research (e.g., [4]-[8]) indicates that the power losses can be reduced by specific torque distribution algorithms, also called control allocation (CA) strategies. For instance, References [4] and [9] present CA strategies minimizing energy dissipation due to tire slip. Although effective, the practical implementation of these strategies requires some form of continuous estimation of the longitudinal and lateral slip velocities of each tire, which is beyond the capability of existing state estimators in normal driving conditions. In [5]-[7] and [10]-[12], the reduction of energy dissipations within the electric motor drives is examined. The presented strategies are mainly based on experimentally measured efficiency maps of electric motors. In particular, Reference [5] carries out an offline calculation of the optimal wheel torques, but without analyzing the resulting wheel torque distribution as a function of the input parameters, i.e., wheel torque demand and vehicle speed. The results in [5] imply that the optimal solution is either to only use a single axle or to evenly distribute the torque among the front and rear drives of the EV. Moreover, the CA strategies in [6], [12], and [13] are shown to be more efficient than the simple even torque distribution among the front and rear axles. However, the influence of vehicle speed $(V)$ on the optimal solution is not directly taken into account. The effect of speed variation is investigated in [7], but it is not formulated. Reference [10] discusses the change in the optimal distribution ratio as a function of longitudinal acceleration. The problem formulation is novel with results indicating small variations in the optimal distribution ratio over the achievable acceleration range. According to the results in [10], the vehicle never operates in the "single-axle" 


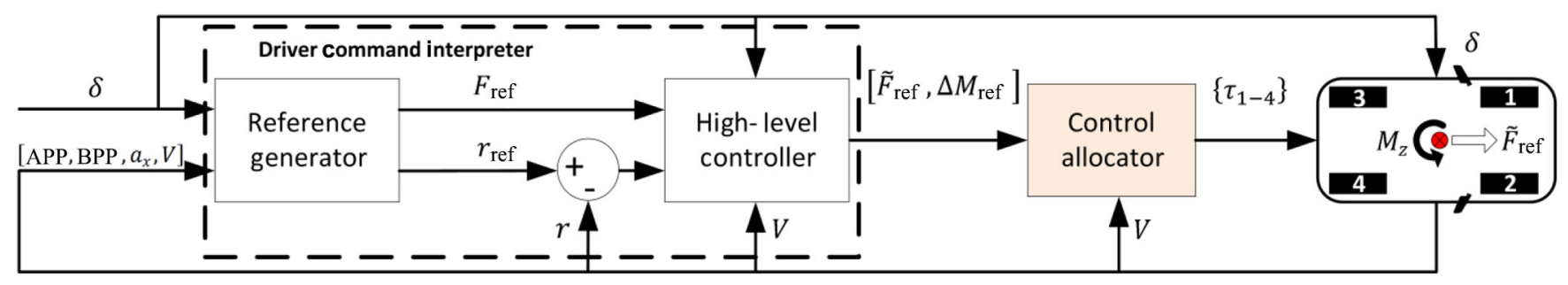

Fig. 1. Vehicle dynamics control structure.

mode, which is shown in [5] and [8] to be the optimal solution for small torque demands. Moreover, Reference [10] only considers straight-line driving. Reference [11] presents a CA strategy that is based on a simplified piecewise-linear efficiency map of the brushless dc motors. An algorithm, based on KarushKuhn-Tucker (KKT) conditions of optimality, finds global solutions of the CA problem for non-monotonically increasing drivetrain power loss curves. The corresponding nonconvex optimization problem is translated into a number of equivalent eigenvalue problems. References [14] and [15] propose high-level controllers aimed at achieving the reference cornering response, coupled with $\mathrm{CA}$ algorithms to improve vehicle stability (e.g., based on tire workload reduction). However, Reference [4] shows that such strategies can be far from optimal in terms of energy efficiency.

In summary, an extensive literature deals with the subject of CA strategies for four-wheel-drive EVs. Many of the proposed algorithms are designed for reduced tire slip variance among the wheels. The papers dealing with drivetrain energy efficiency either do not discuss the resultant wheel torque distribution maps or they present very complicated algorithms (such as multiparametric nonconvex optimization [16], [17]), which are unlikely to be directly implemented on production vehicles. As a consequence, there is a clear need for simple, computationally efficient, easily tunable, and effective solutions of the CA problem aimed at drivetrain energy efficiency. The gap is addressed by this paper, including the following novel contributions:

1) the analytical solution of the CA problem maximizing energy efficiency, under the hypothesis of strictly monotonically increasing drivetrain power losses with wheel torque demand. The optimal solution, obtained for the case of equal drivetrains, is parameterized as a function of $V$;

2) a fast and easily implementable torque distribution strategy maximizing energy efficiency, based on the proposed analytical solution in 1);

3) the simulation-based and experimental validation of the energy benefits of the CA algorithm in cornering conditions and along driving cycles.

This paper is organized as follows. Section II formulates the energy-efficient CA problem as a multiparametric nonconvex optimization. Section III provides the theoretical background required to design the proposed fast CA for EVs. The performance of the CA strategy is verified in Section IV through computer simulations with a vehicle dynamics model and experiments on an electric Range Rover Evoque demonstrator vehicle. Finally, conclusions are provided in Section V.

\section{Problem Statement and Formulation}

\section{A. Problem Statement}

Fig. 1 shows the simplified vehicle control structure. The reference generator outputs the reference yaw rate $r_{\text {ref }}$, and traction/braking force $F_{\text {ref }}$, e.g., starting from the steering wheel angle $(\delta)$, accelerator and brake pedal positions (respectively, APP and BPP), and longitudinal vehicle speed and acceleration (respectively, $V$ and $a_{x}$ ). The high-level controller calculates the corrected longitudinal force reference $\tilde{F}_{\text {ref }}$ (i.e., $F_{\text {ref }}$ from the reference generator is reduced in extreme cornering conditions) and the yaw moment reference $\Delta M_{\text {ref }}$, e.g., based on the combination of feedforward and feedback control of vehicle yaw rate $r$.

The proposed CA strategy must minimize the drivetrain power loss while maintaining $\tilde{F}_{\text {ref }}$ (each drivetrain can operate in either traction or regeneration) and $\Delta M_{\text {ref. }}$. As the drivetrain power losses are functions of $V$, the CA strategy includes $V$ as a parameter.

The CA problem is formulated as a static optimization or quasi-dynamic optimization [18]-[20] to be solved at each time step for the values of $\tilde{F}_{\text {ref }}$ and $\Delta M_{\text {ref }}$ calculated by the highlevel controller, and the estimated $V$ (Fig. 1). The CA strategy could be integrated into the high-level controller, thus giving origin to a single multiple-input multiple-output controller. On the other hand, the separation among the high-level controller and the CA strategy (Fig. 1) has advantages, such as ease of considering actuator limitations [21] and flexibility with respect to the drivetrain configuration. The following section will formulate the CA problem mathematically, under Assumptions 1 and 2 .

Assumption 1: The case study EV includes four identical electric drivetrains with equal power loss characteristics; i.e., the electric machines and their power electronics, single-speed gearboxes, constant-velocity joints, and wheels are the same on each vehicle corner.

Assumption 2: The drivetrain power loss characteristic on each vehicle corner $P_{\text {loss }}\left(\tau_{w}, V\right)$, is positive and strictly monotonically increasing as a function of wheel torque demand $\tau_{w}$. This means that $P_{\text {loss }}\left(\tau_{w}, V\right)>0$ and $\partial P_{\text {loss }}\left(\tau_{w}, V\right) / \partial \tau_{w}>0$.

The results presented in the next paragraph can also be applied (with specific rearrangements) to the simplified case of a four-wheel-drive EV with a single drivetrain per axle. 


\section{B. Mathematical Formulation}

The proposed optimal CA problem for small steering angles is formulated as follows:

$$
\begin{aligned}
& \left\{\boldsymbol{\tau}_{\boldsymbol{w}}\right\}^{*}\left(\tilde{F}_{\mathrm{ref}}, \Delta M_{\mathrm{ref}}, V\right) \\
& \quad=\arg \min _{\tau_{w_{i, t}, \tau_{w_{i, g}}}} J\left(\tau_{w_{i, t}}, \tau_{w_{i, g}}, V\right) \\
& \quad:=\sum_{i=1}^{4}\left[\operatorname{sign}\left(\tau_{w_{i, t}}\right) P_{i, t}\left(\tau_{w_{i, t}}, V\right)\right. \\
& \left.\quad-\operatorname{sign}\left(\tau_{w_{i, g}}\right) P_{i, g}\left(\tau_{w_{i, g}}, V\right)\right] \\
& \text { s.t. } \sum_{i=1}^{4}\left(\tau_{w_{i, t}}-\tau_{w_{i, g}}\right)=\tilde{F}_{\mathrm{ref}} R \\
& \quad d_{f}\left(\tau_{w_{2, t}}+\tau_{w_{1, g}}-\tau_{w_{1, t}}-\tau_{w_{2, g}}\right) \\
& \quad+d_{r}\left(\tau_{w_{4, t}}+\tau_{w_{3, g}}-\tau_{w_{3, t}}-\tau_{w_{4, g}}\right)=\Delta M_{\mathrm{ref}} R \\
& \quad 0 \leq V \leq V_{\max } \\
& \quad 0 \leq \tau_{w_{i, t}} \leq \tau_{w, \max , i, t} \\
& 0 \leq \tau_{w_{i, g}} \leq \tau_{w, \max , i, g} \\
& \tau_{w_{i, t}} \tau_{w_{i, g}}=0 ; i=1,2,3,4
\end{aligned}
$$

In the notation of this paper, $\left\{\boldsymbol{\tau}_{\boldsymbol{w}}\right\}^{*}$ is the vector with the optimal values of the torques, which has different dimensions depending on the specific formulations of the optimization problem presented in this paper. $\tau_{w_{i, t}}$ and $\tau_{w_{i, g}}$ are the traction and regeneration torque demands at the different wheels (numbered as in Fig. 1). $R$ is the tire radius. $d_{f}$ and $d_{r}$ are the front and rear half-tracks. $\tau_{w, \text { max }, i, t}$ and $\tau_{w, \max , i, g}$ are the minimum values between the torque available at the drivetrain in traction, $\tau_{w, \max , t}(V)$, or regeneration, $\tau_{w, \max , g}(V)$, and the transmissible torque at the tire-road contact, $\mu_{x} F_{z, i} R$, being $\mu_{x}$ the estimated tire-road friction coefficient (in longitudinal direction) and $F_{z, i}$ the estimated vertical load at the wheel $i$. The first and second constraints in (1) have a clear physical meaning in terms of vehicle dynamics: they require that the torques applied to the wheels generate, respectively, the corrected longitudinal force reference $\tilde{F}_{\text {ref }}$, and the reference yaw moment $\Delta M_{\text {ref }}$, of the torque-vectoring controller. $\Delta M_{\text {ref }}$ is actuated through different wheel torques on the two sides of the vehicle.

In the hypothesis of low load transfers, (1) has three parameters: 1) $\tilde{F}_{\text {ref }}$;2) $\Delta M_{\text {ref }}$; and 3) $V$. The problem is nonconvex due to the complementarity constraints and nonconvexities in the power loss characteristics of the electric machines and mechanical transmission systems. The complementarity constraints $\tau_{w_{i, t}} \tau_{w_{i, g}}=0$ specify that at each instant, each wheel can only operate in traction or regeneration. For the same reason, the sign function in (1) allows to take only either $P_{i, t}$ or $P_{i, g}$ (note that $\left.\operatorname{sign}(0)=0\right) . P_{i, t}$ and $P_{i, g}$ are, respectively, the drawn and regenerated electrical powers at the wheel $i$, given by

$$
\begin{aligned}
P_{i, t} & =\tau_{w_{i, t}} \frac{V}{R}+P_{\text {loss }, t}\left(\tau_{w_{i, t}}, V\right) ; i=1,2,3,4 \\
P_{i, g} & =\tau_{w_{i, g}} \frac{V}{R}-P_{\text {loss }, g}\left(\tau_{w_{i, g}}, V\right) ; i=1,2,3,4
\end{aligned}
$$

where the first term on the right-hand side is the mechanical power at the wheel and the second term is the power loss. The power losses of all vehicle corners are modeled with the same functions $P_{\text {loss }, t / g}$ (the subscripts indicate traction and regeneration, respectively) obtained by fitting the experimental measurements on the drivetrains to a mathematical expression (Assumption 1). In general, the power loss characteristics are different from zero at zero wheel torque demand. This is the reason for the sign function in (1).

By replacing (2) into the cost function $J\left(\tau_{w_{i, t}}, \tau_{w_{i, g}}, V\right)$ of (1), $J$ is reformulated as

$$
\begin{aligned}
J\left(\tau_{w_{i, t}}, \tau_{w_{i, g}}, V\right)= & \frac{V}{R}(\overbrace{\sum_{i=1}^{4}\left(\tau_{w_{i, t}}-\tau_{w_{i, g}}\right)}^{\tilde{F}_{\text {ref } R}}) \\
& +\sum_{i=1}^{4}\left[\operatorname{sign}\left(\tau_{w_{i, t}}\right) P_{\text {loss }, t}\left(\tau_{w_{i, t}}, V\right)\right. \\
& \left.+\operatorname{sign}\left(\tau_{w_{i, g}}\right) P_{\text {loss }, g}\left(\tau_{w_{i, g}}, V\right)\right]
\end{aligned}
$$

where the first term on the right-hand side is the overall mechanical power at the wheels and the second term is the overall power loss.

The term $(V / R) \tilde{F}_{\text {ref }} R$ is constant for given values of the parameters. Hence, the cost function can be reduced to

$$
\begin{aligned}
J\left(\tau_{w_{i, t}}, \tau_{w_{i, g}}, V\right)= & \sum_{i=1}^{4}\left[\operatorname{sign}\left(\tau_{w_{i, t}}\right) P_{\text {loss }, t}\left(\tau_{w_{i, t}}, V\right)\right. \\
& \left.+\operatorname{sign}\left(\tau_{w_{i, g}}\right) P_{\text {loss }, g}\left(\tau_{w_{i, g}}, V\right)\right]
\end{aligned}
$$

Equation (4) assumes that the vehicle operates with limited yaw rate and limited relative slip among the wheels, which means that $V$ can be used as the speed of each vehicle corner.

In summary, (1) is a multiparametric nonlinear programming (mp-NLP) problem [22], [23], with the following general formulation:

$$
\begin{aligned}
& z(\boldsymbol{\theta})=\min _{x \in X} J(x, \boldsymbol{\theta}) \\
& \text { s.t. } G(x, \boldsymbol{\theta}) \leq 0 ; \quad H(x, \boldsymbol{\theta})=0 ; \quad \boldsymbol{\theta} \in \Theta
\end{aligned}
$$

where $\boldsymbol{\theta}=\left[\tilde{F}_{\text {ref }}, \Delta M_{\text {ref }}, V\right]^{T}$ is the parameter vector, and $G$ and $H$ represent the inequality and equality constraints.

\section{ili. Controller Design}

Lemma 1: Problem (1) has only one solution for each side of the vehicle, assuming $d=d_{f}=d_{r}$ :

$$
\begin{aligned}
& \tau_{w, l}^{*}=0.5\left(\tilde{F}_{\text {ref }}-\frac{\Delta M_{\mathrm{ref}}}{d}\right) R \\
& \tau_{w, r}^{*}=0.5\left(\tilde{F}_{\mathrm{ref}}+\frac{\Delta M_{\mathrm{ref}}}{d}\right) R \\
& -\tau_{w, \max , l, g} \leq \tau_{w, l}^{*} \leq \tau_{w, \max , l, t} \\
& -\tau_{w, \max , r, g} \leq \tau_{w, r}^{*} \leq \tau_{w, \max , r, t}
\end{aligned}
$$


where $\tau_{w, l}^{*}$ and $\tau_{w, r}^{*}$ are, respectively, the optimal wheel torque demands of the left- and right-hand sides of the vehicle, with $\tau_{w, \max , l, t / g}=\tau_{w, \max , 1, t / g}+\tau_{w, \max , 3, t / g}$ and $\tau_{w, \max , r, t / g}=$ $\tau_{w, \max , 2, t / g}+\tau_{w, \max , 4, t / g}$ (the subscripts ' $t$ ' and ' $g$ ' indicate traction and regeneration, respectively).

Proof: From the vehicle schematic in Fig. 1

$$
\begin{aligned}
\tau_{w, l} & =\tau_{w_{1, t}}+\tau_{w_{3, t}}-\tau_{w_{1, g}}-\tau_{w_{3, g}} \\
\tau_{w, r} & =\tau_{w_{2, t}}+\tau_{w_{4, t}}-\tau_{w_{2, g}}-\tau_{w_{4, g}}
\end{aligned}
$$

where $\tau_{w, l}$ and $\tau_{w, r}$ are, respectively, the wheel torque demands of the left- and right-hand sides of the vehicle.

By replacing (4) and (7) into (1), (1) becomes

$$
\begin{gathered}
\left\{\boldsymbol{\tau}_{\boldsymbol{w}}\right\}^{*}\left(\tilde{F}_{\mathrm{ref}}, \Delta M_{\mathrm{ref}}, V\right) \\
=\arg \min _{\tau_{w, l}, \tau_{w, r}} J\left(\tau_{w, l}, \tau_{w, r}, V\right) \\
:=P_{\operatorname{loss}_{l}}\left(\tau_{w, l}, V\right)+P_{\operatorname{loss}_{r}}\left(\tau_{w, r}, V\right) \\
\text { s.t. } \tau_{w, l}+\tau_{w, r}=\tilde{F}_{\text {ref }} R \\
\tau_{w, r}-\tau_{w, l}=\frac{\Delta M_{\text {ref }} R}{2 d} \\
0 \leq V \leq V_{\max } \\
-\tau_{w, \max , l, g} \leq \tau_{w, l} \leq \tau_{w, \max , l, t} \\
-\tau_{w, \max , r, g} \leq \tau_{w, r} \leq \tau_{w, \max , r, t}
\end{gathered}
$$

where $P_{\operatorname{loss}_{l}}$ and $P_{\operatorname{loss}_{r}}$ are the power losses of the drivetrains on the left and right vehicle sides. The complementarity conditions $\tau_{w_{i, t}} \tau_{w_{i, g}}=0$ are no longer present in (8) as they are internal conditions for the left- and right-hand sides; this implies that $\tau_{w, l}$ and $\tau_{w, r}$ can only vary between $-\tau_{w, \max , l / r, g}$ and $\tau_{w, \max , l / r, t}$ (the subscripts ' $l$ ' and ' $r$ ' indicate left and right, respectively). Problem (8) has a unique solution, as in (6), due to the two equality constraints for the decision variables $\tau_{w, l}$ and $\tau_{w, r}$. The solution is fixed and independent of the cost function.

Remark 1: The solutions for the left- and right-hand sides of the vehicle are independent of $V$ and only depend on $\tilde{F}_{\text {ref }}$ and $\Delta M_{\text {ref }}$.

Remark 2: The reference values of the total longitudinal force and yaw moment are restricted, in first approximation, by the maximum and minimum torques of the electric motors (which depend on $V$ ) and, in second approximation, by the estimated tire-road friction coefficient.

Remark 3: As mentioned before, because of the two equality constraints with two decision variables in the main problem, a unique analytical feasible solution exists. To consider torque rate constraints, these two equality constraints need to be relaxed or transferred to the cost function to make the problem feasible.

Lemma 2: If Assumptions 1 and 2 hold, the optimal torque distributions for the left- or right-hand sides of the vehicle make both front and rear motors work in either traction or regeneration (including the case that one motor is switched off and only one motor is producing torque).

Proof: Lemma 1 proves that (1) can be simplified to an optimal torque distribution problem for each side of the vehicle. The optimal torque distribution of the left-hand side of the vehicle for the case of traction, i.e., $\tau_{w, l}^{*} \geq 0$, is the solution of the following problem:

$$
\begin{aligned}
& \left\{\boldsymbol{\tau}_{\boldsymbol{w}}\right\}^{*}\left(\tau_{w, l}^{*}, V\right) \\
& =\arg \min _{\tau_{w_{1, t}}, \tau_{w_{3, t}}, \tau_{w_{1, g}}, \tau_{w_{3, g}}} \operatorname{sign}\left(\tau_{w_{1, t}}\right) P_{\text {loss }, \mathrm{t}}\left(\tau_{w_{1, t}}, V\right) \\
& +\operatorname{sign}\left(\tau_{w_{3, \mathrm{t}}}\right) P_{\text {loss }, \mathrm{t}}\left(\tau_{w_{3, \mathrm{t}}}, V\right) \\
& +\operatorname{sign}\left(\tau_{w_{1, g}}\right) P_{\text {loss }, g}\left(\tau_{w_{1, g}}, V\right) \\
& +\operatorname{sign}\left(\tau_{w_{3, g}}\right) P_{\text {loss }, g}\left(\tau_{w_{3, g}}, V\right) \\
& \text { s.t. } \tau_{w_{1, t}}+\tau_{w_{3, \mathrm{t}}}-\tau_{w_{1, g}}-\tau_{w_{3, g}}=\tau_{w, l}^{*} \\
& \tau_{w_{i, t}} \tau_{w_{i, g}}=0 ; i \in\{1,3\} \\
& 0 \leq \tau_{w_{i, t}} \leq \tau_{w, \max , i, t} \\
& 0 \leq \tau_{w_{i, g}} \leq \tau_{w, \max , i, g}
\end{aligned}
$$

The same problem is defined for the right-hand side of the vehicle or for the regeneration case, i.e., $\tau_{w, l}^{*}<0$. Expanding the complementarity constraint of (9), the optimal solution of (9) is the best solution among the following four problems:

$$
\begin{aligned}
& \left\{\boldsymbol{\tau}_{\boldsymbol{w}}\right\}^{*}\left(\tau_{w, l}^{*}, V\right) \\
& =\arg \min _{\tau_{w_{1}}, \tau_{w_{3}}} P_{\text {loss }, t / g}\left(\tau_{w_{1}}, V\right)+P_{\text {loss }, t / g}\left(\tau_{w_{3}}, V\right) \\
& \text { s.t. }\left\{\begin{array}{l}
\tau_{w_{1}}+\tau_{w_{3}}=\tau_{w, l}^{*} ; \text { or } \\
-\tau_{w_{1}}-\tau_{w_{3}}=\tau_{w, l}^{*} ; \text { or } \\
\tau_{w_{1}}-\tau_{w_{3}}=\tau_{w, l}^{*} ; \text { or } \\
-\tau_{w_{1}}+\tau_{w_{3}}=\tau_{w, l}^{*} .
\end{array}\right. \\
& 0 \leq \tau_{w_{i}} \leq \tau_{w, \max , i, t} \text { or } \\
& 0 \leq \tau_{w_{i}} \leq \tau_{w, \max , i, g} ; i \in\{1,3\}
\end{aligned}
$$

where $\tau_{w_{1}}$ and $\tau_{w_{3}}$ are the torques at the left front and left rear wheels, i.e., $\tau_{w_{i}}=\tau_{w_{i, t}}$ in traction or $\tau_{w_{i}}=\tau_{w_{i, g}}$ in regeneration. $\tau_{w_{1}}+\tau_{w_{3}}=\tau_{w, l}^{*}$ and $-\tau_{w_{1}}-\tau_{w_{3}}=\tau_{w, l}^{*}$ represent the pure traction and regeneration cases, while $\tau_{w_{1}}-$ $\tau_{w_{3}}=\tau_{w, l}^{*}$ and $-\tau_{w_{1}}+\tau_{w_{3}}=\tau_{w, l}^{*}$ are the cases in which one of the wheels is in traction and the other one in regeneration.

The resulting value of the cost function in (10) is smaller without regeneration, as $P_{\text {loss }}$ is positive and strictly monotonically increasing, i.e., $P_{\text {loss }} \geq 0$ and $\partial P_{\text {loss }} / \partial \tau_{w}>0$ (Assumption 2). Any regeneration increases the absolute value of the traction torque and, therefore, according to (2), increases the total power loss. As a result, the conditions $\tau_{w_{1}}-\tau_{w_{3}}=$ $\tau_{w, l}^{*}$ and $-\tau_{w_{1}}+\tau_{w_{3}}=\tau_{w, l}^{*}$ are not optimal cases and, depending on the sign of $\tau_{w, l}^{*}$, both wheels on the same side must work in either traction or regeneration.

Theorem 1: Suppose that Assumptions 1 and 2 hold, $P_{\text {loss }}\left(\tau_{w}\right)$ has the shape indicated in Fig. 2 (with a nonconvex region followed by a convex region, i.e., with a single saddle point) and there are no torque rate constraints; then, for each side of the vehicle:

a) single axle is the optimal solution for small values of the torque demand;

b) even distribution is the optimal solution for large values of the torque demand; 


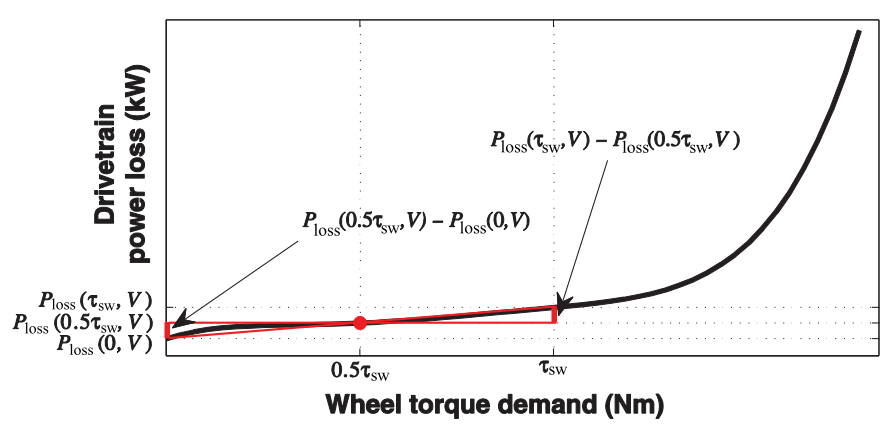

Fig. 2. Geometrical interpretation of Theorem 1, indicating the optimal switching point on the drivetrain power loss characteristic.

c) the optimal switching point between solutions 1) and 2) at vehicle speed $V$ can be calculated as the solution of

$$
P_{\text {loss }, t / g}\left(\tau_{\text {sw }}, V\right)+P_{\text {loss }, t / g}(0, V)=2 P_{\text {loss }, t / g}\left(0.5 \tau_{\text {sw }}, V\right)
$$

where $\tau_{\mathrm{sw}}$ is the switching torque.

Proof: Lemma 2 proves that the optimal torque distribution of the left-hand side of the vehicle with $\tau_{w, l}^{*} \geq 0$ is the solution of the following problem:

$$
\begin{aligned}
\left\{\boldsymbol{\tau}_{w}\right\}^{*}\left(\tau_{w, l}^{*}, V\right)= & \arg \min _{\tau_{w_{1}}, \tau_{w_{3}}} J\left(\tau_{w_{1}}, \tau_{w_{3}}, V\right) \\
& :=P_{\text {loss }, t}\left(\tau_{w_{1}}, V\right)+P_{\text {loss }, t}\left(\tau_{w_{3}}, V\right) \\
\text { s.t. } \tau_{w_{1}}+\tau_{w_{3}} & =\tau_{w, l}^{*} \\
0 \leq \tau_{w_{i}} & \leq \tau_{w, \max , i, t} ; i \in\{1,3\}
\end{aligned}
$$

By approximating the cost function of (12) around $\tau_{w, 0}=$ $\tau_{w, l}^{*} / 2$, which is the solution of the even distribution strategy, the Taylor series is

$$
\begin{aligned}
& J\left(\tau_{w_{1}}, \tau_{w_{3}}, V\right) \stackrel{=}{=} \\
& P_{\text {loss }, t}\left(\tau_{w, 0}, V\right)+\nabla_{\tau_{w}} P_{\text {loss }, t}\left(\tau_{w, 0}, V\right)\left(\tau_{w_{1}}-\tau_{w, 0}\right) \\
& +\frac{1}{2} \nabla_{\tau_{w} \tau_{w}}^{2} P_{\text {loss }, t}\left(\tau_{w, 0}, V\right)\left(\tau_{w_{1}}-\tau_{w, 0}\right)^{2} \\
& +P_{\text {loss }, t}\left(\tau_{w, 0}, V\right)+\nabla_{\tau_{w}} P_{\text {loss }, t}\left(\tau_{w, 0}, V\right)\left(\tau_{w_{3}}-\tau_{w, 0}\right) \\
& +\frac{1}{2} \nabla_{\tau_{w} \tau_{w}}^{2} P_{\text {loss }, t}\left(\tau_{w, 0}, V\right)\left(\tau_{w_{3}}-\tau_{w, 0}\right)^{2}
\end{aligned}
$$

Since $\tau_{w_{1}}+\tau_{w_{3}}=\tau_{w, l}^{*}$ is a constant value, if $\tau_{w_{1}}$ (or $\tau_{w_{3}}$ ) deviates from $\tau_{w, 0}$ by the amount $\varepsilon$, then $\tau_{w_{3}}$ (or $\tau_{w_{1}}$ ) must deviate from $\tau_{w, 0}$ by the same amount but in opposite direction

$$
\tau_{w_{1}}=\tau_{w, 0}+\varepsilon ; \quad \tau_{w_{3}}=\tau_{w, 0}-\varepsilon
$$

By substituting (14) into (13), the Taylor series approximation in (13) is reformulated as

$$
\begin{aligned}
& J(\varepsilon, V) \cong 2 P_{\text {loss }, t}\left(\tau_{w, 0}, V\right)+\nabla_{\tau_{w} \tau_{w}}^{2} P_{\text {loss }, t}\left(\tau_{w, 0}, V\right) \varepsilon^{2} \\
& \quad \text { s.t. }-\tau_{w, 0} \leq \varepsilon \leq \tau_{w, 0}
\end{aligned}
$$

$2 P_{\text {loss }}\left(\tau_{w, 0}, V\right)$ is the power loss of the even distribution case. Therefore, for $P_{\text {loss }} \geq 0$, the following holds:

1) If the Hessian of $P_{\text {loss }}$ is negative, i.e., $P_{\text {loss }}$ is nonconvex at $\tau_{w, 0}$, then $J(\varepsilon, V) \leq 2 P_{\text {loss }}\left(\tau_{w, 0}, V\right)$ and, therefore, the even distribution is the worst strategy. In this case, the minimum value of the cost function is achieved with the biggest offset from $\tau_{w, 0}$. From Lemma 2 (see also Fig. 2), the biggest offset is for the case $|\varepsilon|=\tau_{w, 0}$, and therefore, $\tau_{w_{1}}$ or $\tau_{w_{3}}$ must be zero, which is equivalent to the single-axle strategy a);

2) If the Hessian of $P_{\text {loss }}$ is positive or zero at $\tau_{w, 0}$, i.e., $P_{\text {loss }}$ is convex at $\tau_{w, 0}$, then $J(\varepsilon, V) \geq 2 P_{\text {loss }}\left(\tau_{w, 0}, V\right)$, which means that the even distribution $(\varepsilon=0)$ is the optimal solution. Fig. 2 shows that $P_{\text {loss }}$ is typically convex for large torque demands, and therefore, 2) is proven.

A switching torque $\tau_{\mathrm{sw}}$ between these two strategies can be found, i.e., a torque value exists at which the power loss of the even distribution strategy $2 P_{\text {loss }}\left(0.5 \tau_{\mathrm{sw}}, V\right)$, is equal to the one of the single-axle strategy $P_{\text {loss }}\left(\tau_{\text {sw }}, V\right)+P_{\text {loss }}(0, V)$.

Fig. 2 represents a geometrical interpretation of the switching torque $\tau_{\text {sw }}$. It shows that the single-axle strategy is the optimal one up to the point at which $P_{\text {loss }}\left(\tau_{w}, V\right)-P_{\text {loss }}(0, V)=$ $P_{\text {loss }}\left(2 \tau_{w}, V\right)-P_{\text {loss }}\left(\tau_{w}, V\right)$. The left- and right-hand sides of the inequality are, respectively, the power loss saving by switching off one of the drivetrains (i.e., $\tau_{w, 1 / 3}=0$ ), and the power loss increases by the other drivetrain, when switching from even distribution to single axle. If Assumption 2) holds, these two terms become equal at the switching torque $\tau_{\mathrm{sw}}$.

Remark 4: Based on Theorem 1, the optimal distribution strategy for $\tau_{w, l}^{*} \geq 0$ is as follows:

$$
\begin{aligned}
& \tau_{w, 1}^{*}\left(\tau_{w, l}^{*}, V\right)= \begin{cases}\tau_{w, l}^{*}, & \tau_{w, l}^{*} \leq \tau_{\mathrm{sw}}(V) \\
0.5 \tau_{w, l}^{*}, & \tau_{w, l}^{*}>\tau_{\mathrm{sw}}(V)\end{cases} \\
& \tau_{w, 3}^{*}\left(\tau_{w, l}^{*}, V\right)= \begin{cases}0, & \tau_{w, l}^{*} \leq \tau_{\mathrm{sw}}(V) \\
0.5 \tau_{w, l}^{*}, & \tau_{w, l}^{*}>\tau_{\mathrm{sw}}(V)\end{cases}
\end{aligned}
$$

where $\tau_{w, 1}^{*}$ and $\tau_{w, 3}^{*}$ are, respectively, the optimal torque demands of the front and rear wheels of the left-hand side of the vehicle. In the single-axle strategy, the front motors are selected (instead of the rear motors) for safety reasons; in fact, in limited conditions, it is preferable to have understeer rather than oversteer.

The equivalent right-hand side torques are calculated with the same approach. In the practical implementation of the controller, a sigmoid function is used to approximate the discontinuity of (16), to prevent drivability issues deriving from the fast variation in the torque demands.

Remark 5: $\tau_{\mathrm{sw}}$ depends on the value of $V$. Since the problem is parametric, the solution is parametric as well. In practice, $V$ can be estimated with a suitable Kalman filter using the four wheel speeds and the longitudinal acceleration of the vehicle. References [24] and [25] describe methods for vehicle speed estimation.

Remark 6: If $P_{\text {loss }}\left(\tau_{w}\right)$ is convex (i.e., without the saddle point in Fig. 2), then the even distribution is the optimal solution for any torque demand.

Remark 7: If (13) is not a good approximation of $J\left(\tau_{w_{1}}, \tau_{w_{3}}, V\right)$, the even-order derivatives of $P_{\text {loss }, t}\left(\tau_{w}, V\right)$ with respect to $\tau_{w}$ must either be zero or have the same sign for a given value of $\tau_{w}$. For example, this is the case if $P_{\text {loss }, t}\left(\tau_{w}, V\right)$ is a cubic polynomial. The experimental power 


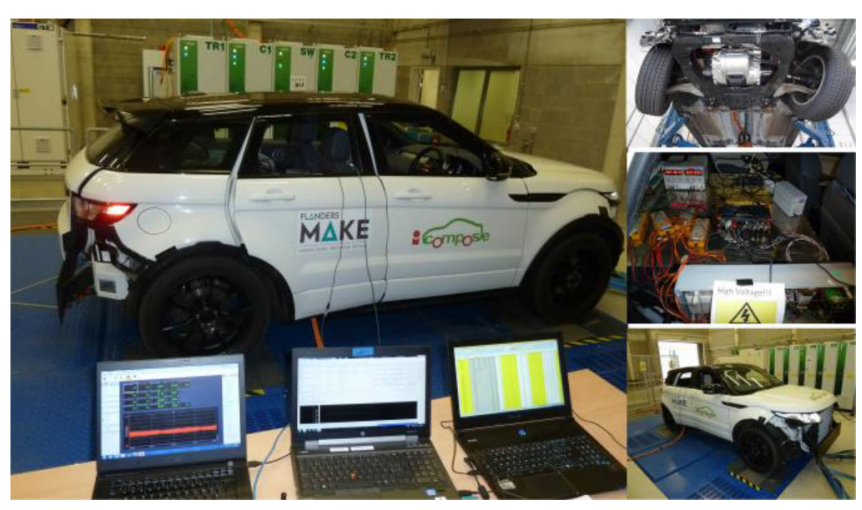

Fig. 3. Range Rover Evoque setup on the rolling road facility at Flanders MAKE (Belgium).

TABLE I

MAIN VehicLe PARAmeters

\begin{tabular}{clc}
\hline \hline Symbol & \multicolumn{1}{c}{ Name and dimension } & Value \\
\hline$\ell$ & Wheelbase $(\mathrm{m})$ & 2.665 \\
$\tau_{\mathrm{gb}}$ & Gearbox ratio $(-)$ & 10.56 \\
$R$ & Wheel radius (m) & 0.364 \\
$d$ & Half-track (m) & 0.808 \\
- & Number of motors per axle $(-)$ & 2 \\
$V_{\mathrm{dc}}$ & High-voltage dc bus level $(\mathrm{V})$ & 600 \\
$T_{n}$ & Motor nominal torque $(\mathrm{Nm})$ & 80 \\
$P_{n}$ & Motor nominal power $(\mathrm{kW})$ & 35 \\
$P_{p}$ & Motor peak power $(\mathrm{kW})$ & 75 \\
\hline \hline
\end{tabular}

loss characteristics reported in Section IV are well interpolated by a cubic polynomial.

Remark 8: The developed CA strategy is overruled in two cases:

1) In the case of wheel torque saturation (see Section II-B), the torque demand beyond the limit is transferred to the other wheel on the same side until saturation is reached as well;

2) For significant braking, an electronic braking distribution (EBD) strategy intervenes to maintain the correct relative slip ratio among the tires of the front and rear axles.

\section{Results}

\section{A. Experiments}

1) Experimental System Setup: Fig. 3 shows the experimental setup for the validation of the developed torque distribution strategy. The vehicle demonstrator is an electric Range Rover Evoque with four identical on-board switched reluctance motors, connected to the wheels through singlespeed transmissions, constant-velocity joints, and half-shafts. Table I reports the main vehicle parameters.

A dSPACE AutoBox system is used for running all controllers (Fig. 1), including the reference generator, the highlevel controller (as in [26]), and the CA algorithm. The electrical power is provided by an external supply (visible in the background of Fig. 3), which is connected in parallel with the battery pack. Therefore, the high-voltage dc bus level is maintained steady around $600 \mathrm{~V}$.

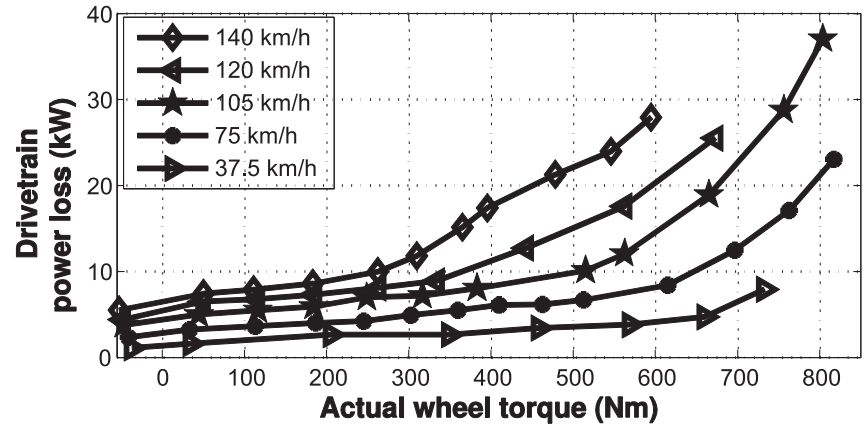

Fig. 4. Power losses of a single drivetrain, measured on the rolling road facility.

The tests were conducted using a MAHA rolling road facility (located at Flanders MAKE, Belgium) allowing speed and torque control modes of the rollers. In the speed control mode, the roller speeds are constantly kept at the specified set value irrespective of variations in the actual wheel torques. The torque demand at each wheel is assigned manually through the dSPACE interface, and therefore, the vehicle can be tested for any assigned achievable torque demand and speed. The rig includes the measurement of the longitudinal force and speed of the rollers, and thus, allows the evaluation of the overall drivetrain efficiency, i.e., from the electrical power at the inverters to the mechanical power at the rollers during traction (opposite flow in the case of regeneration). In the torque control mode, the roller bench applies a torque to the rollers, which emulates tire rolling resistance, aerodynamic drag, and vehicle inertia. Therefore, the torque control mode is used for driving cycle testing. The vehicle follows the reference velocity profile of the specific driving schedule through a velocity trajectory tracker (i.e., a model of the human driver) implemented on the dSPACE system as the combination of a feedforward and feedback controller, providing a wheel torque demand output.

2) Drivetrain Power Loss Measurement: The experimental drivetrain power loss characteristics of the case study EV are reported in Fig. 4 for a number of vehicle speeds. The power loss curves are plotted in terms of the actual wheel torque. The first point of each curve corresponds to the case of zero wheel torque demand, resulting in nonzero power loss mainly due to tire rolling resistance; conversely, the actual wheel torque is zero when the torque supplied by the drivetrain compensates rolling resistance.

Fig. 4 shows that the drivetrain power loss characteristics are positive and strictly monotonically increasing functions of wheel torque, hence confirming Assumption 2). The curves are nonconvex for low wheel torques and become convex for large torque values. Therefore, Theorem 1 is applicable to the design of an energy-efficient CA algorithm for the electric Range Rover Evoque demonstrator. Fig. 5 reports the measured efficiencies of each side of the EV demonstrator in terms of the front-to-total torque ratio, for different values of $V$ and side torque demands. The relative slip among the wheels does not change significantly for the different combinations of speeds, torque demands, and front-to-total torque ratios. Due to the nonconvexity of the power loss at low torque demands, the 


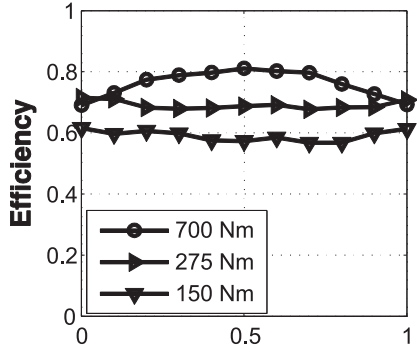

Front-to-total torque ratio

(a)

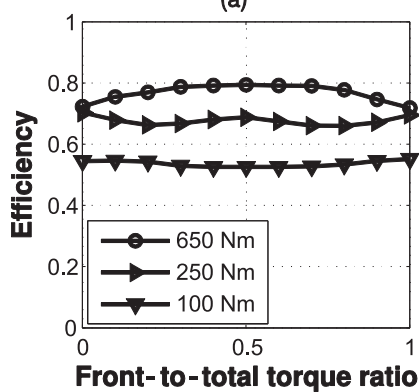

(c)

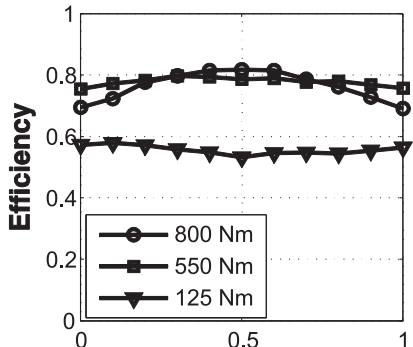

Front-to-total torque ratio

(b)

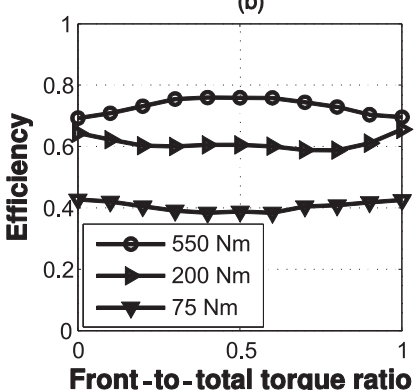

(d)
Fig. 5. Measured drivetrain efficiencies as a function of the front-to-total torque ratio, for different side torques at vehicle speeds of: (a) $40 \mathrm{~km} / \mathrm{h}$, (b) $65 \mathrm{~km} / \mathrm{h}$, (c) $90 \mathrm{~km} / \mathrm{h}$, and (d) $115 \mathrm{~km} / \mathrm{h}$.

efficiency is higher for torque ratios of 0 or 1 , i.e., for the singleaxle strategy. In contrast, an even distribution (corresponding to the torque ratio of 0.5 ) is the optimal solution for high torque demands. As a result, the optimal torque distribution on each side of the vehicle is achieved by switching between the single axle and even distribution strategies depending on torque demand and vehicle speed.

3) Switching Torque Calculation: Based on the measured power loss curves in Fig. 4, the switching torque $\tau_{s w}$, is calculated using Theorem 1 and (11). The power loss curve at each vehicle speed is piecewise linearly interpolated to construct $P_{\text {loss }}\left(\tau_{w}, V\right)$. The method assumes that the tire rolling resistance power losses are equal at each vehicle corner, which is a reasonable approximation in normal driving conditions with low values of the load transfers (which directly affect tire power losses). The longitudinal slip power losses are included in the rolling road measurement. The method assumes that the slip ratios are similar to those measured on the rolling road, which is also a reasonable approximation in normal driving conditions. Knowing $P_{\text {loss }}\left(\tau_{w}, V\right),(11)$ is solved offline with an exhaustive search method to calculate $\tau_{\mathrm{sw}}$. Fig. 6 shows the calculated $\tau_{\text {sw }}$ on each side of the vehicle for different $V$. The values were stored as a look-up table in the controller running on the dSPACE system. In terms of implementation on the vehicle, the developed procedure is fast, i.e., it can easily be run in real time on hardware with low-computational processing power.

4) Driving Cycle Tests: To assess the energy efficiency benefits of the developed CA strategy in straight-line conditions, three different driving cycles were performed with the vehicle demonstrator on the rolling road. The driving cycles are the New European Driving Cycle (NEDC), the Artemis Road driving cycle, and the Extra Urban Driving Cycle (EUDC) with the hypothesis of a constant $8 \%$ (uphill) slope of the road.

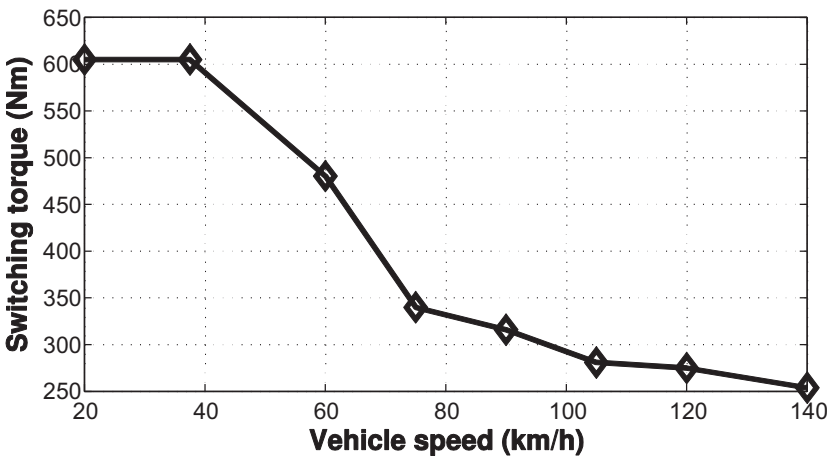

Fig. 6. Switching torque $\left(\tau_{\mathrm{sw}}\right)$ for each side of the vehicle as a function of $V$.

TABLE II

Energy Consumption OVer Different DRIVING CyCLes

\begin{tabular}{|c|c|c|c|c|c|}
\hline \multirow{2}{*}{$\begin{array}{l}\text { Driving } \\
\text { cycle }\end{array}$} & \multicolumn{3}{|c|}{ Energy consumptions (kWh) } & \multicolumn{2}{|c|}{$\begin{array}{c}\text { CA improvements (\%) } \\
\text { with respect to }\end{array}$} \\
\hline & $\begin{array}{l}\text { Single- } \\
\text { axle }\end{array}$ & $\begin{array}{c}\text { Even } \\
\text { distribution }\end{array}$ & $\begin{array}{l}\text { With } \\
\text { CA }\end{array}$ & $\begin{array}{l}\text { Single- } \\
\text { axle }\end{array}$ & $\begin{array}{c}\text { Even } \\
\text { distribution }\end{array}$ \\
\hline NEDC & 2.921 & 3.059 & 2.918 & 0.1 & 4.6 \\
\hline $\begin{array}{l}\text { Artemis- } \\
\text { road }\end{array}$ & 4.487 & 4.634 & 4.442 & 1.0 & 4.1 \\
\hline $\begin{array}{c}\text { EUDC 8\% } \\
\text { slope }\end{array}$ & 5.793 & 5.740 & 5.709 & 1.5 & 0.5 \\
\hline
\end{tabular}

The energy consumption of the vehicle with the CA strategy was measured and compared with the ones for the fixed single axle and even distribution strategies. Table II shows the measurement results, which indicate energy savings of up to $\sim 5 \%$ for the developed CA algorithm, depending on the driving cycle. Interestingly, the NEDC and Artemis Road favor the single-axle strategy over the even distribution because of the relatively low wheel torque demand. This trend is reversed with the EUDC with $8 \%$ slope, as it is a more aggressive cycle with mostly high torque demands. The fact that for some conditions, the specific vehicle is more efficient with the single-axle strategy is caused by the significant values of the peak power and torque of its electric drivetrains, which work at very low demands during conventional driving cycles.

The operation of the developed torque distribution algorithm over a segment of a driving cycle is investigated in Fig. 7, which confirms the validity of the CA strategy. For instance, between 305 and $309 \mathrm{~s}$, when the vehicle speed is $\sim 117 \mathrm{~km} / \mathrm{h}$, the side torque demand is $\sim 215 \mathrm{Nm}$, i.e., less than the calculated switching torque of $280 \mathrm{Nm}$ in Fig. 6, and therefore the single-axle strategy is the optimal solution, as in Fig. 7(a).

\section{B. Simulations}

To examine the performance of the CA torque distribution strategy in cornering conditions, ramp steer simulations with a validated CarMaker/Simulink vehicle model (see details in [26]) were performed. For the simulations, the vehicle was accelerated to $V=110 \mathrm{~km} / \mathrm{h}$ and then a constant steering wheel rate of $3 \%$ and up to $100^{\circ}$ was applied.

Fig. 8 depicts the simulated longitudinal wheel forces as functions of lateral acceleration $a_{y}$, according to the developed CA strategy. The single-axle strategy is the solution for both sides of the vehicle for lateral accelerations below $2.6 \mathrm{~m} / \mathrm{s}^{2}$. 

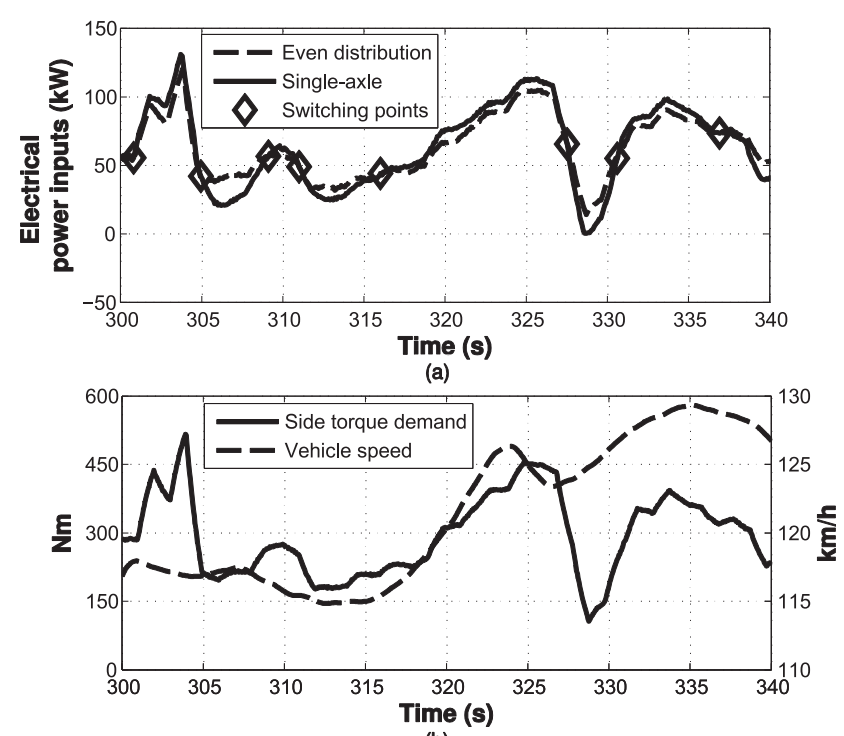

(b)

Fig. 7. Sample of driving cycle measurements: (a) electrical power input for the even distribution and single-axle strategies with the switching points indicated by markers; (b) side torque demands overlapped with $V$.

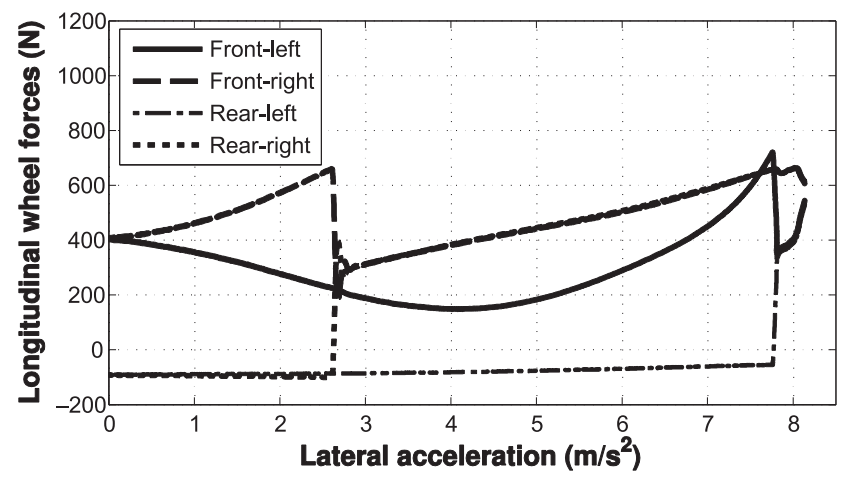

Fig. 8. Longitudinal wheel forces, simulated during the ramp steer maneuver at $100 \mathrm{~km} / \mathrm{h}$ with the developed CA strategy. The outer and inner sides switch from single axle to even distribution, respectively, at $a_{y}$ of 2.6 and $7.8 \mathrm{~m} / \mathrm{s}^{2}$.

For $a_{y}>7.8 \mathrm{~m} / \mathrm{s}^{2}$, the solution for both sides is the even distribution. Between the two $a_{y}$ values, the switching between the strategies is dependent on the side of the vehicle - namely, with the increasing $a_{y}$, the outer side of the vehicle switches earlier to even distribution due to the yaw moment and its contribution to the longitudinal forces in (6). This happens because the specific high-level controller setup (see Section II-A) applies a yaw moment which reduces vehicle understeer, i.e., the torque demand (the parameter on which the switching is actually based) on the outer side of the car is higher than on the inner side. Therefore, when the lateral acceleration is between 2.6 and $7.8 \mathrm{~m} / \mathrm{s}^{2}$, the torque demand is distributed equally between the front and rear wheels on the outer side and is applied only to the front wheel on the inner side of the vehicle. In other words, three motors are simultaneously active.

Fig. 9 shows the total vehicle power loss (excluding the aerodynamic losses) simulated for the three different torque distribution strategies: 1) even distribution; 2) single axle; and 3 ) the developed CA strategy. As with the experimental tests,

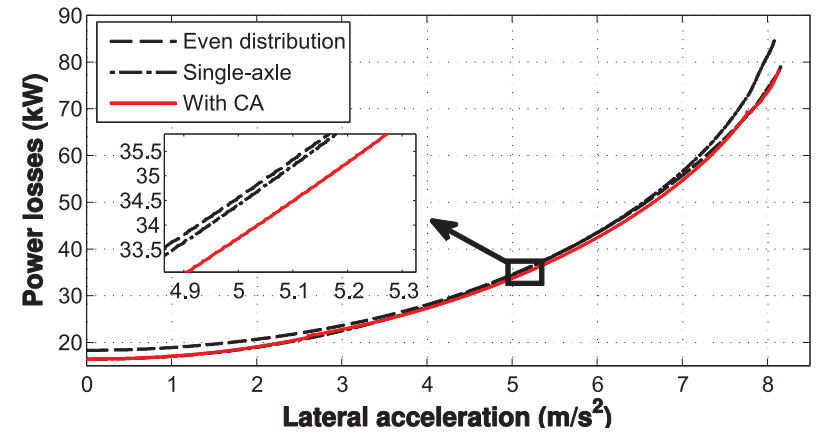

Fig. 9. Total drivetrain power losses (including tire power losses) simulated during the ramp steer maneuver for even distribution, single axle, and the developed CA strategy.

the CA strategy reduces the drivetrain power losses here by up to $10 \%$ and $8 \%$ compared to the even distribution and single-axle strategies. The biggest improvements are achieved at very low and very high lateral accelerations where, respectively, the single-axle strategy and the even distribution strategy are the optimal solutions (see also Fig. 6). For lateral accelerations between 2.6 and $7.8 \mathrm{~m} / \mathrm{s}^{2}$, when the optimal solution for the outer and inner vehicle sides are different (Fig. 8), the developed CA strategy reduces the power loss by $\sim 2.4 \%$ (Fig. 9).

\section{CONCLUSION}

The presented research work allows the following conclusions, essential for the design of a fast, energy-efficient, and easily implementable torque allocation algorithm for fourwheel-drive EVs with equal drivetrains on the front and rear axles.

1) For relatively small values of steering wheel angle, the CA problem of a four-wheel-drive EV with multiple motors can be independently solved for the left- and right-hand sides of the vehicle.

2) If the power loss characteristics of the electric drivetrains are strictly monotonically increasing functions of the torque demand, the minimum consumption is achieved using a single motor on each side of the vehicle up to a torque demand threshold, and an even torque distribution among the front and rear motors above such threshold.

3) An analytical formula is proposed for the computation of the torque demand threshold, which is a function of vehicle speed, based on the drivetrain power loss characteristic.

4) The developed strategy is easily implementable as a small-sized look-up table in the main control unit of the vehicle, allowing real-time operation with minimum demand on the processing hardware.

5) The experimental analysis of the case study drivetrain efficiency characteristics as functions of the front-to-total wheel torque distribution confirms the validity of the proposed CA algorithm at different vehicle velocities and torque demands, i.e., at low torques, the single axle is the optimal solution, and at high torques, the even distribution is the optimal solution. 
6) The experimental results for a four-wheel-drive EV demonstrator along driving cycles show energy consumption reductions between $0.1 \%$ and $5 \%$, with respect to the same vehicle with single axle or even torque distribution strategies.

7) The simulation results of ramp steer maneuvers indicate significant energy consumption reductions for the whole range of lateral accelerations.

The approximation related to the assumptions of tire slip ratios similar to those in the rolling road experiments and negligible rolling resistance variation with vertical load will be addressed in future work.

\section{REFERENCES}

[1] Y. Wang, B.M. Nguyen, H. Fujimoto, and Y. Hori, "Multirate estimation and control of body sideslip angle for electric vehicles based on onboard vision system," IEEE Trans. Ind. Electron., vol. 61, no. 2, pp. 1133-1143, Feb. 2014.

[2] K. Nam, S. Oh, and H. Fujimoto, "Estimation of sideslip and roll angles of electric vehicles using lateral tire force sensors through RLS and Kalman filter approaches," IEEE Trans. Ind. Electron., vol. 60, no. 3, pp. 9881000, Mar. 2013.

[3] A. Pennycott, L. De Novellis, P. Gruber, and A. Sorniotti, "Sources of energy loss during torque-vectoring for fully electric vehicles," Int. J. Veh. Des., vol. 67, no. 2, pp. 157-177, 2014.

[4] Y. Suzuki, Y. Kano, and M. Abe, "A study on tyre force distribution controls for full drive-by-wire electric vehicle," Veh. Syst. Dyn., vol. 52, pp. 235-250, 2014.

[5] R. Wang, Y. Chen, D. Feng, X. Huang, and J. Wang, "Development and performance characterization of an electric ground vehicle with independently actuated in-wheel motors," J. Power Sources, vol. 196, pp. 3962-3971, 2011.

[6] Y. Chen and J. Wang, "Adaptive energy-efficient control allocation for planar motion control of over-actuated electric ground vehicles," IEEE Trans. Control Syst. Technol., vol. 22, no. 4, pp. 1362-1373, Jul. 2014.

[7] A. Pennycott, L. De Novellis, A. Sabbatini, P. Gruber, and A. Sorniotti, "Reducing the motor power losses of a four-wheel drive, fully electric vehicle via wheel torque allocation," Proc. Inst. Mech. Eng., J. Automobile Eng., vol. 228, no. 7, pp. 830-839, 2014.

[8] X. Yuan and J. Wang, "Torque distribution strategy for a front- and rearwheel-driven electric vehicle," IEEE Trans. Veh. Technol., vol. 61, no. 8, pp. 3365-3374, Oct. 2012.

[9] L. De Novellis, A. Sorniotti, and P. Gruber, "Wheel torque distribution criteria for electric vehicles with torque-vectoring differentials," IEEE Trans. Veh. Technol., vol. 63, no. 4, pp. 1593-1602, May 2014.

[10] H. Fujimoto and S. Harada, "Model-based range extension control system for electric vehicles with front and rear driving-braking force distributions," IEEE Trans. Ind. Electron., vol. 62, no. 5, pp. 3245-3254, May 2015.

[11] Y. Chen and J. Wang, "Fast and global optimal energy-efficient control allocation with applications to over-actuated electric ground vehicles," IEEE Trans. Control Syst. Technol., vol. 20, no. 5, pp. 1202-1211, Sep. 2012.

[12] Y. Chen and J. Wang, "Design and experimental evaluations on energy efficient control allocation methods for overactuated electric vehicles: Longitudinal motion case," IEEE/ASME Trans. Mechatronics, vol. 19, no. 2, pp. 538-548, Apr. 2014.

[13] Y. Chen and J. Wang, "Energy-efficient control allocation with applications on planar motion control of electric ground vehicles," in Proc. Amer. Control Conf., 2011, pp. 2719-2724.

[14] S. Fallah, A. Khajepour, B. Fidan, C. Shih-Ken, and B. Litkouhi, "Vehicle optimal torque vectoring using state-derivative feedback and linear matrix inequality," IEEE Trans. Veh. Technol., vol. 62, no. 4, pp. 1540-1552, May 2013.

[15] B. Li, A. Goodarzi, A. Khajepour, S.K. Chen, and B. Litkouhi, "An optimal torque distribution control strategy for four-independent wheel drive electric vehicles," Veh. Syst. Dyn., vol. 53, no. 8, pp. 1-18, 2015.

[16] P. Tøndel and T. A. Johansen, "Control allocation for yaw stabilization in automotive vehicles using multiparametric nonlinear programming," in Proc. Amer. Control Conf., 2005, pp. 453-458.
[17] T. A. Johansen, T. P. Fuglseth, P. Tøndel, and T. I. Fossen, "Optimal constrained control allocation in marine surface vessels with rudders," Control Eng. Pract., vol. 16, no. 4, pp. 457-464, 2008.

[18] J. Tjønnås and T. A. Johansen, "Adaptive control allocation," Automatica, vol. 44 , no. 11 , pp. $2754-2765,2008$.

[19] J. Tjonnas and T. A. Johansen, "Stabilization of automotive vehicles using active steering and adaptive brake control allocation," IEEE Trans. Control Syst. Technol., vol. 18, no. 3, pp. 545-558, May 2010.

[20] T. A. Johansen and T. I. Fossen, "Control allocation-A survey," Automatica, vol. 49, no. 5, pp. 1087-1103, 2013.

[21] O. Härkegård and S. T. Glad, "Resolving actuator redundancy-Optimal control vs. control allocation," Automatica, vol. 41, no. 1, pp. 137-144, 2005.

[22] L. F. Domínguez, D. A. Narciso, and E. N. Pistikopoulos, "Recent advances in multiparametric nonlinear programming," Comput. Chem. Eng., vol. 34, no. 5, pp. 707-716, 2010.

[23] A. Grancharova and T. A. Johansen, "Multi-parametric programming," in Explicit Nonlinear Model Predictive Control - Theory and Applications. New York, NY, USA: Springer, 2012, vol. 429, pp. 1-37.

[24] U. Kiencke and L. Nielsen, Automotive Control Systems: For Engine, Driveline, and Vehicle. New York, NY, USA: Springer, 2005.

[25] M. Tanelli, S. M. Savaresi, and C. Cantoni, "Longitudinal vehicle speed estimation for traction and braking control systems," in Proc. IEEE Int. Conf. Control Appl., 2006, pp. 2790-2795.

[26] L. De Novellis et al., "Direct yaw moment control actuated through electric drivetrains and friction brakes: Theoretical design and experimental assessment," Mechatronics, vol. 26, pp. 1-15, 2015.

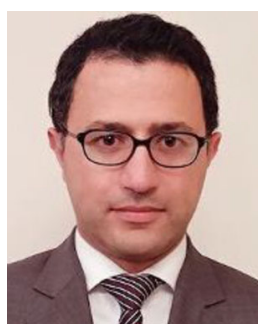

Arash M. Dizqah (M'12) received the B.Sc. degree in electrical engineering from Sharif University of Technology, Tehran, Iran, in 1998, the M.Sc. degree in electrical engineering from K.N. Toosi University of Technology, Tehran, Iran, in 2001, and the Ph.D. degree in control engineering from Northumbria University, Newcastle upon Tyne, U.K., in 2014.

He was a Research Fellow with the University of Surrey, Guildford, U.K. He is a Lecturer of Intelligent Transport Systems with Coventry University, Coventry, U.K. His research interests include control and optimization, and nonlinear model-predictive controllers for connected vehicles, electric and hybrid vehicles, and renewable energy systems.

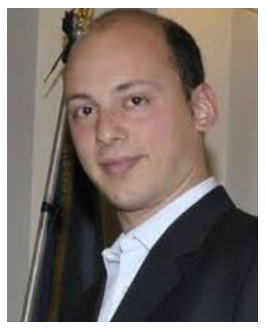

Basilio Lenzo (M'13) received the M.Sc. degree in mechanical engineering from the University of Pisa and the Scuola Superiore Sant'Anna, Pisa, Italy, in 2010, and the Ph.D. degree in robotics from the Scuola Superiore Sant'Anna in 2013.

In 2010, he was an R\&D Intern with Ferrari F1, Maranello, Italy. In 2013, he was a Visiting Researcher at the University of Delaware, Newark, DE, USA, and Columbia University, New York, NY, USA. In 2013, he was appointed as a Research Fellow with the Scuola Superiore Sant'Anna, where he worked on kinematics and dynamics of robotic mechanisms. Since 2015, he has been a Research Fellow with the Centre for Automotive Engineering, University of Surrey, Guildford, U.K. His research interests include vehicle dynamics, simulation, control, and robotics.

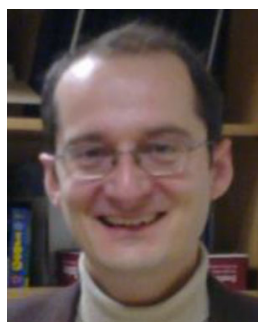

Aldo Sorniotti (M'12) received the M.Sc. degree in mechanical engineering and the Ph.D. degree in applied mechanics from the Politecnico di Torino, Turin, Italy, in 2001 and 2005, respectively.

$\mathrm{He}$ is a Reader of Advanced Vehicle Engineering with the University of Surrey, Guildford, U.K., where he is the Coordinator of the Centre for Automotive Engineering. His research interests include vehicle dynamics control, integration of chassis control systems, and transmission systems for electric and hybrid electric vehicles. 


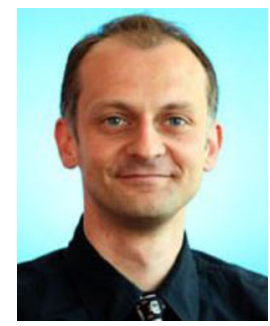

Patrick Gruber received the M.Sc. degree in motorsport engineering and management from Cranfield University, Cranfield, U.K., in 2005, and the Ph.D. degree in mechanical engineering from the University of Surrey, Guildford, U.K., in 2009.

$\mathrm{He}$ is a Senior Lecturer of Advanced Vehicle Systems Engineering with the University of Surrey. His research interests include tire dynamics and development of novel tire models.

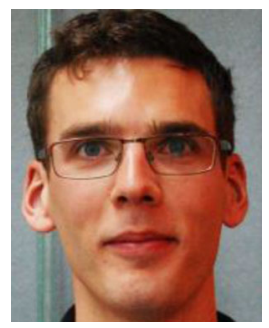

Jasper De Smet received the M.Sc. degree in electrical engineering from the Katholieke Universiteit (KU) Leuven (Campus De Nayer), Sint-Katelijne-Waver, Belgium, in 2008.

After graduation, he had a one-year internship with Eurocopter, Marignane, France. Since 2009, he has been a Researcher with Flanders MAKE, Lommel, Belgium. His research interests include integration and control of electromechanical systems in vehicles and machines.

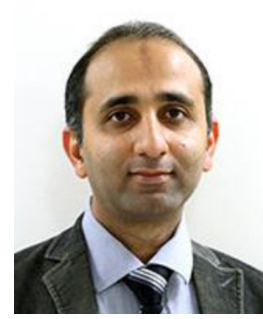

Saber Fallah received the B.Sc. degree from Isfahan University of Technology, Isfahan, Iran, in 2001, the M.Sc. degree from Shiraz University, Shiraz, Iran, in 2004, and the Ph.D. degree from Concordia University, Montreal, QC, Canada, in 2010, all in mechanical engineering.

$\mathrm{He}$ is a Lecturer with the University of Surrey, Guildford, U.K. His research interests include vehicle dynamics and control, electric and hybrid electric vehicles, intelligent vehicles, and vehicle system design and integration. 